\title{
Sorafenib and Palbociclib Combination Regresses a Cisplatinum-resistant Osteosarcoma in a PDOX Mouse Model
}

\author{
TAKASHI HIGUCHI ${ }^{1,2,3}$, NORIHIKO SUGISAWA ${ }^{1,2}$, KENTARO MIYAKE $^{1,2}$, \\ HIROMICHI OSHIRO ${ }^{1,2}$, NORIO YAMAMOTO ${ }^{3}$, KATSUHIRO HAYASHI ${ }^{3}$, HIROAKI KIMURA ${ }^{3}$, \\ SHINJI MIWA ${ }^{3}$, KENTARO IGARASHI $^{3}$, SANT P. CHAWLA $^{4}$, MICHAEL BOUVET ${ }^{2}$, \\ SHREE RAM SINGH ${ }^{5}$, HIROYUKI TSUCHIYA ${ }^{3}$ and ROBERT M. HOFFMAN ${ }^{1,2}$ \\ ${ }^{1}$ AntiCancer, Inc., San Diego, CA, U.S.A.; \\ ${ }^{2}$ Department of Surgery, University of California, San Diego, CA, U.S.A.; \\ ${ }^{3}$ Department of Orthopedic Surgery, Kanazawa University, Kanazawa, Japan; \\ ${ }^{4}$ Sarcoma Oncology Center, Santa Monica, CA, U.S.A.; \\ ${ }^{5}$ Basic Research Laboratory, National Cancer Institute, Frederick, MD, U.S.A.
}

\begin{abstract}
Background/Aim: Recurrent osteosarcoma is a recalcitrant disease; therefore, an improved strategy is urgently needed to provide therapy. In order to develop a novel strategy for this disease, our lab has developed a patient-derived orthotopic xenograft (PDOX) mouse model for osteosarcoma. The combination of sorafenib (SFN) and palbociclib (PAL) was shown to be effective of hepatocellular carcinoma. However, whether this combination is efficacious on osteosarcoma has not been reported. The aim of this study was to determine the efficacy of the SFN and PAL combination on a cisplatinum (CDDP)-resistant osteosarcoma PDOX model. Materials and Methods: Osteosarcoma PDOX models were randomly divided into five treatment groups: untreated-control, $C D D P, S F N, P A L$ and the combination of SFN and PAL. Results: Of these agents, the SFN-PAL combination significantly regressed tumor growth, and enhanced tumor necrosis with degenerative changes in the osteosarcoma PDOX. Conclusion: The SFN-PAL combination is an effective treatment strategy for osteosarcoma and therefore holds promise for clinical efficacy.
\end{abstract}

Correspondence to: Robert M. Hoffman, 7917 Ostrow St., San Diego, CA 92111, U.S.A. Tel: +1 8586542555, e-mail: all@anticancer.com; Shree Ram Singh, Basic Research Laboratory, National Cancer Institute, 1050 Boyles St., Frederick, MD, 21702, U.S.A. Tel: +1 3018467331, e-mail: singhshr@mail.nih.gov; Hiroyuki Tsuchiya, Department of Orthopaedic Surgery, Kanazawa University, 13-1, Takara-machi, Kanazawa 920-8641, Japan. Tel: +81 0762652374, email: tsuchi@med.kanazawa-u.ac.jp

Key Words: Osteosarcoma, sorafenib, palbociclib, PDOX, combination therapy.
Cisplatinum (CDDP) is widely used against osteosarcoma (1). However, recurrent or advanced osteosarcoma is a recalcitrant disease resistant to CDDP and other first-line therapies (2). Sorafenib (SFN) is an inhibitor of several tyrosine protein kinases that has been approved for the treatment of various cancers (3-5). Palbociclib (PAL) is a cyclin-dependent kinase $4 / 6$ (CDK4/6) inhibitor that regulates the cell-cycle, inhibits cell growth and suppresses DNA replication (6-8).

CDK inhibitors are preferentially used in combination with other drugs that convert temporary cell-cycle inhibition to permanent growth arrest or cell death (7). SFN and PAL have been shown to have synergistic effects against hepatocellular carcinoma and pancreatic carcinoma $(6,7)$. However, the efficacy of SFN and PAL combination for osteosarcoma has not been reported.

In the present report, using a patient-derived orthotopic xenograft (PDOX) model of osteosarcoma, we demonstrate for the first time that the SFN-PAL combination could overcome CDDP-resistance.

\section{Materials and Methods}

Mice. Athymic nu/nu nude mice (AntiCancer, Inc., San Diego, CA, USA) were used in this study $(9,10)$. Detail protocols for animal handing, breeding, anesthesia and surgery have been presented previously (9-13). All experiments were performed at AntiCancer, Inc. The protocols used in the mouse study were approved by the Institutional Animal Care and Use Committee (IACUC) (9). All the studies were conducted according to the principles and procedures described in the National Institute of Health Guide for the Care and Use of Animals under Assurance Number A3873-1 (9).

Patient-derived tumor. A fresh biopsy sample from a 14-year-old boy with pelvic conventional osteosarcoma was used (10). Sample preparation and its subcutaneous implantation in nude mice has been previously described (10). Before biopsy this patient did not 


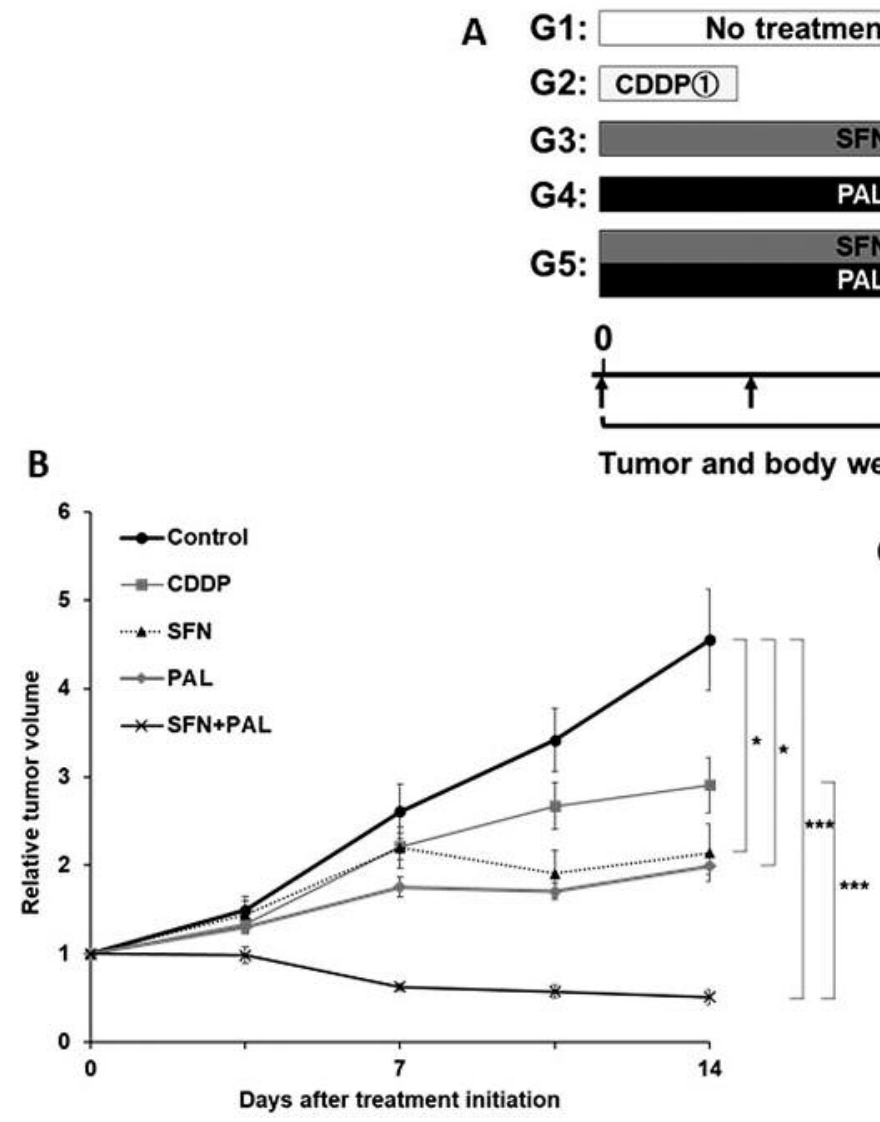

CDDP(2)

G3: SFN, daily

G4: PAL, daily

G5:

FN, daily

AL, daily

B

Figure 1. Treatment schema and efficacy of drugs. (A) Treatment protocols in the osteosarcoma PDOX model. (B) Relative tumor volume of the osteosarcoma-PDOX model. The relative tumor volume is the tumor volume at any time during treatment compared to the tumor volume at the initiation of treatment. Untreated control ( $p=0.56)$; SFN ( $p=0.02)$; PAL $(p=0.01)$; and SFN-PAL $(p<0.001)$. (C) Waterfall plot of relative tumor volume (day $14 /$ day 0 ) for each mouse. $N=6$ mice/group. ${ }^{*} p<0.05$; *** $p<0.001$. Error bars: \pm SEM. receive any chemotherapy or radiotherapy. The patient has provided a written informed consent as part of a UCLA Institutional Review Board approved protocol (IRB\#10-001857) (11).

Developing the osteosarcoma PDOX model. The detailed procedures for mouse anesthesia, and orthotopic implantation of tumors into the mouse distal femur have been previously described $(12,13)$. The detailed protocols for skin incision and tumor fragment implantation were performed as described previously $(12,13)$.

Treatment protocols in the osteosarcoma PDOX model. The osteosarcoma PDOX mouse models were randomly divided into five groups. Each group contained six mice. The mice were treated with the following drugs for two weeks (Figure 1A) as previously described (10): G1-untreated control; G2-CDDP (6 $\mathrm{mg} / \mathrm{kg}$, intraperitoneal (i.p.) injection, once a week) alone; G3-SFN (30 mg/kg, oral, daily) alone; G4-PAL (100 mg/kg, oral, daily); G5-SFN + PAL. Treatment started when all tumors reached $100 \mathrm{~mm}^{3}$ volume. Tumor length, width, volume, and mouse body weight were measured as described previously (10). Data are presented as mean \pm standard error of the mean (SEM).

Histological analysis. Fixation of fresh tumor samples, sectioning and staining were performed as described previously (10).
Ethical approval. The study was performed under AntiCancer Inc. IACUC (Institutional Animal Care and Use Committee)-approved protocol. The patient has provided a written informed consent as part of a UCLA Institutional Review Board approved protocol (IRB\#10-001857) (11).

Statistical analysis. All statistical analyses were performed as described previously (10). The Shapiro-Wilk test was used for normal distribution (10). To verify the homogeneity of variances across groups, the Bartlett's test was performed. For the parametric test for inter-group comparison, one-way ANOVA with Tukey HSD for post-hoc analysis was performed (10) and to compare the means between two related groups, the paired $t$-test was performed (10). All $p$-values were two sided and $p$-values of less than 0.05 were regarded as statistically significant.

\section{Results}

Testing efficacy of drugs on the osteosarcoma-PDOX. Osteosarcoma PDOX models treated with CDDP showed no difference in comparison to untreated controls $(p=0.56)$. While SFN alone or PAL alone significantly, but moderately, 

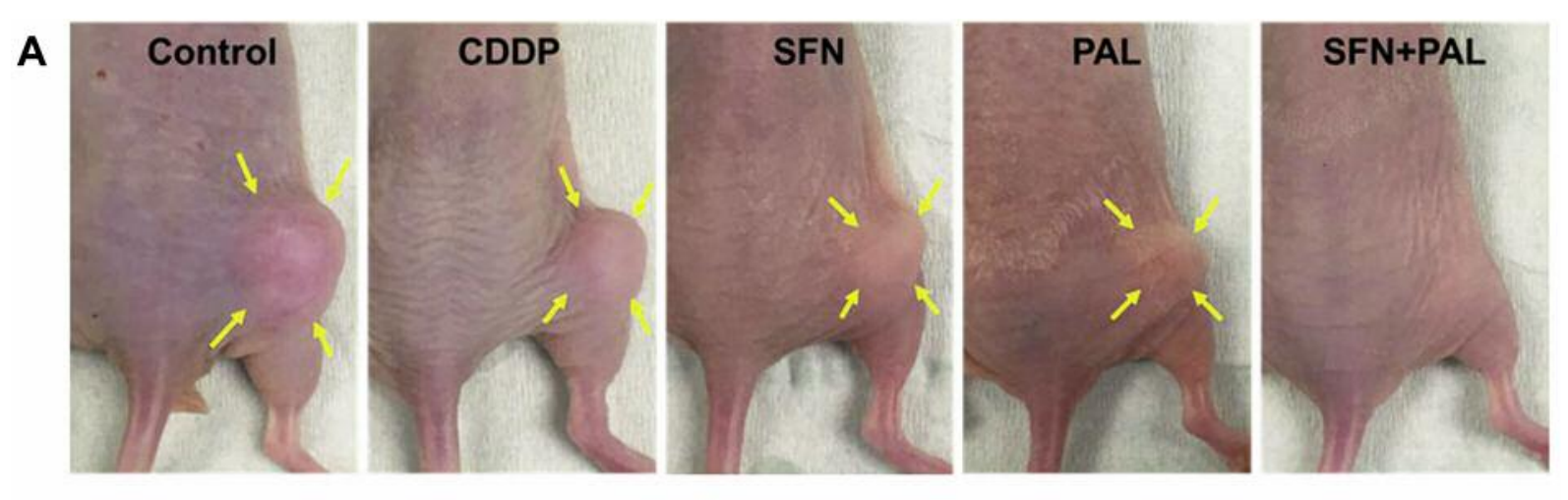

B

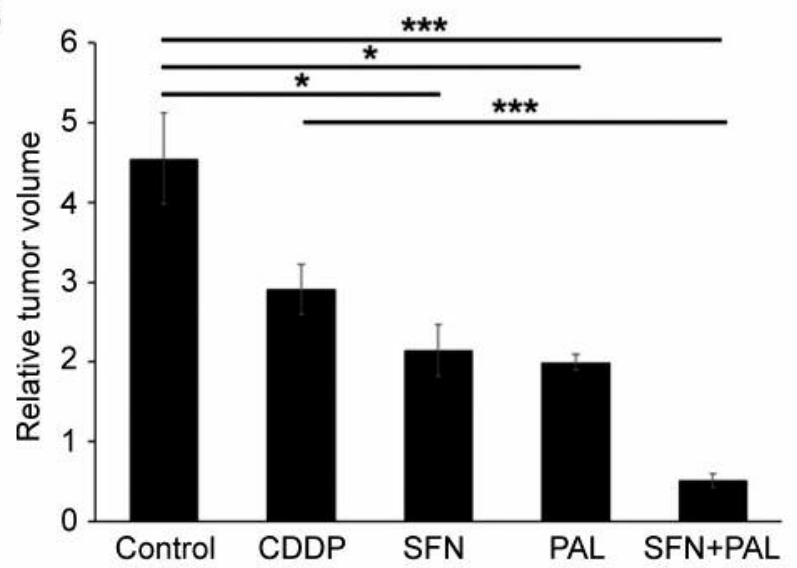

Figure 2. Efficacy of drugs on tumor volume. (A) Representative images of osteosarcoma PDOX mouse models at the end of each treatment. Arrows indicate the clear margin of the tumors. (B) Bar graphs show relative tumor volume of each treatment group on day 14. N=6 mice/group. * $p<0.05$; $* * * p<0.001$. Error bars: \pm SEM.

inhibited the growth of osteosarcoma PDOX compared to the untreated control $(p=0.02$ and $p=0.01$, respectively). In contrast, the combination of SFN-PAL dramatically regressed the osteosarcoma PDOX tumor $(p<0.001)$. Of these drugs, only the SFN-PAL combination was more efficacious compared to CDDP $(p<0.001)$. (Figures 1B, C and $2 \mathrm{~A}, \mathrm{~B})$.

Histology of the osteosarcoma PDOX. Histological analysis showed that the untreated control tumor had viable highlydense cancer cells that were pleomorphic and spindleshaped. Untreated control tumors also had many mitotic events and osteoid laid down in a lace-like pattern among tumor cells (10). Tumors treated with a single dose of CDDP, SFN, or PAL had viable tumor cells, although the cancer-cell density was relatively low as compared to the untreated control. However, tumors treated with the SFN-PAL combination presented cancer necrosis with non-viable cells and frequent occurrence of degenerative scars in the stroma. These results suggested that this combination is efficacious in the osteosarcoma PDOX models (Figure 3A).
Effect of treatment on body weight. As compared to initial body weight, in the untreated control group, the final body weight of mice was significantly increased $(p=0.02)$. However, no significant difference in body weight among the other groups was noted (Figure 3B). In addition, no other observable side effects or animal death in any group were noted.

\section{Discussion}

Here, for the first time, we found that the SFN-PAL combination regressed tumor in an osteosarcoma PDOX model. The synergistic effect of the SFN-PAL combination treatment in cancer treatment has been investigated only in hepatocellular and pancreatic carcinomas $(6,7)$, but not in osteosarcoma. SFN and PAL alone have shown potential efficacy for osteosarcoma $(3,4,14)$. Most osteosarcomas have upregulated VEGFR, PDGFR, or MAPK activation, and inhibition of MAPK by SFN has been shown to be highly effective in osteosarcoma preclinical models (4). It has been shown that CDK4 is highly expressed in osteosarcoma tissues and cell lines (14). An elevated CDK4 expression in immunohistochemical analysis of 

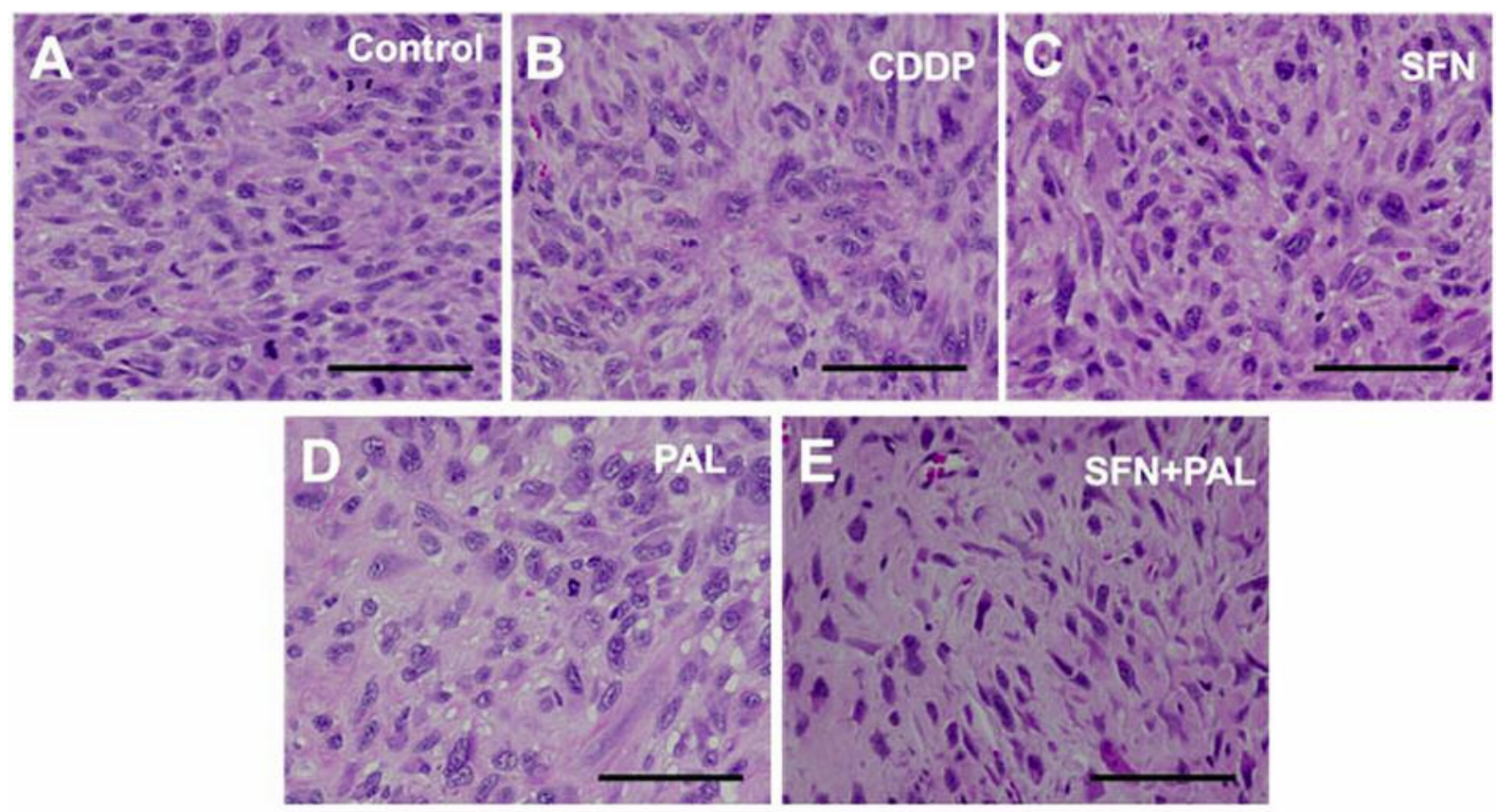

$\mathbf{F}$

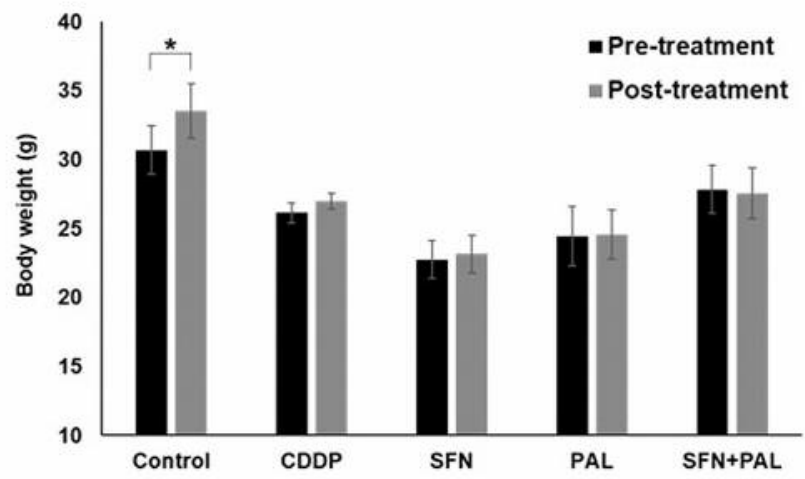

Figure 3. Tumor histology and mouse body weight. (A) Untreated control, (B) CDDP, (C) SFN, (D) PAL, and (E) SFN + PAL. Scale bars: $100 \mu$ m. (F) Bar graphs show mouse body weight in untreated-control and each treatment group at pre- and post-treatment times. ${ }^{*} p<0.05$. Error bars: \pm SEM.

osteosarcoma patients correlated with the development of metastasis and poor prognosis, suggesting that PAL may be effective for osteosarcoma (14). Here, we showed the moderate efficacy of SFN and PAL as single usage, and the high efficacy of the SFN-PAL combination on the cisplatinum-resistant osteosarcoma PDOX model for the first time. The ability of SFN-PAL combination to regress PDOX tumor suggests clinical efficacy.

Our laboratory developed PDOX mouse models of various cancers (9-13, 15-19). Previously, we have shown that our PDOX model is more patient-like compared to subcutaneous patient-derived xenograft (PDX) models $(15,16)$. In addition, we have demonstrated that the PDOX model maintains the original histological and molecular features after xenograft in nude mise $(17,18)$. PDOX models provide the opportunity to test unique personalized treatment options for sarcoma patients.

In conclusion, this study identified an effective treatment strategy using the SFN-PAL combination for recalcitrant osteosarcoma which holds promise of clinical efficacy.

\section{Conflicts of Interest}

AntiCancer Inc. uses PDOX models for contract research. TH, NS, MK, $\mathrm{HO}, \mathrm{NY}, \mathrm{KH}, \mathrm{HK}, \mathrm{SM}, \mathrm{KI}$ and RMH are or were unsalaried associates of AntiCancer Inc. There are no other competing financial interests.

\section{Authors' Contributions}

Conception and design: TH and RMH. Acquisition of data: TH, NS, $\mathrm{KM}, \mathrm{HO}, \mathrm{NM}, \mathrm{KH}, \mathrm{HK}, \mathrm{SM}$, and KI. Analysis and interpretation of 
data: TH, NS, KM, HO, NM, KH, HK, SM, KI, SPC, MB, SRS, HT, and RMH. Writing, review, and/or revision of the manuscript: $\mathrm{TH}, \mathrm{RMH}, \mathrm{HT}$, and SRS.

\section{Acknowledgements}

This paper is dedicated to the memory of Reese Imhoff.

\section{References}

1 Jaffe N: Osteosarcoma: review of the past, impact on the future. The American experience. Cancer Treat Res 152: 239-262, 2009. PMID: 20213394. DOI: 10.1007/978-1-4419-0284-9_12

2 Miwa S, Takeuchi A, Ikeda H, Shirai T, Yamamoto N, Nishida H, Hayashi K, Tanzawa Y, Kimura H, Igarashi K and Tsuchiya $\mathrm{H}$ : Prognostic value of histological response to chemotherapy in osteosarcoma patients receiving tumor-bearing frozen autograft. PloS One 8(8): e71362, 2013. PMID: 23977028. DOI: 10.1371/ journal.pone.0071362

3 Grignani G, Palmerini E, Dileo P, Asaftei SD, D’Ambrosio L, Pignochino Y, Mercuri M, Picci P, Fagioli F, Casali PG, Ferrari $\mathrm{S}$ and Aglietta M: A phase II trial of sorafenib in relapsed and unresectable high-grade osteosarcoma after failure of standard multimodal therapy: an Italian Sarcoma Group study. Ann Oncol 23(2): 508-516, 2012. PMID: 21527590. DOI: 10.1093/annonc/ mdr 151

4 Coventon J: A review of the mechanism of action and clinical applications of sorafenib in advanced osteosarcoma. J Bone Oncol 8: 4-7, 2017. PMID: 28828294. DOI: 10.1016/j.jbo. 2017.07.001

5 Dal Lago L, D'Hondt V and Awada A: Selected combination therapy with sorafenib: a review of clinical data and perspectives in advanced solid tumors. Oncologist 13(8): 845858, 2008. PMID: 18695262. DOI: 10.1634/theoncologist.20070233

6 Lee T, Kim K, Lee J, Park SH, Park YS, Lim HY, Kang WK, Park JO and Kim ST: Antitumor activity of sorafenib plus CDK4/6 inhibitor in pancreatic patient derived cell with KRAS mutation. J Cancer 9(18): 3394-3399, 2018. PMID: 30271501. DOI: $10.7150 /$ jca. 26068

7 Bollard J, Miguela V, Ruiz de Galarreta M, Venkatesh A, Bian CB, Roberto MP, Tovar V, Sia D, Molina-Sánchez P, Nguyen CB, Nakagawa S, Llovet JM, Hoshida Y and Lujambio A: Palbociclib (PD-0332991), a selective CDK4/6 inhibitor, restricts tumour growth in preclinical models of hepatocellular carcinoma. Gut 66(7): 1286-96, 2017. PMID: 27849562. DOI: 10.1136/gutjnl-2016-312268

8 Dickson MA, Schwartz GK, Keohan ML, D'Angelo SP, Gounder MM, Chi P, Antonescu CR, Landa J, Qin LX, Crago AM, Singer S, Koff A and Tap WD: Progression-free survival among patients with well-differentiated or dedifferentiated liposarcoma treated with CDK4 inhibitor palbociclib: A Phase 2 clinical trial. JAMA Oncol 2(7): 937-940, 2016. PMID: 27124835. DOI:10.1001/jamaoncol.2016.0264

9 Higuchi T, Kawaguchi K, Miyake K, Han Q, Tan Y, Oshiro H, Sugisawa N, Zhang Z, Razmjooei S, Yamamoto N, Hayashi K, Kimura H, Miwa S, Igarashi K, Chawla SP, Singh AS, Eilber FC, Singh SR, Tsuchiya H and Hoffman RM: Oral recombinant methioninase combined with caffeine and doxorubicin induced regression of a doxorubicin-resistant synovial sarcoma in a PDOX mouse model. Anticancer Res 38(10): 5639-5644, 2018. PMID: 30275182. DOI: 10.21873/anticanres.12899

10 Higuchi T, Miyake K, Oshiro H, Sugisawa N, Yamamoto N, Hayashi K, Kimura H, Miwa S, Igarashi K, Chawla SP, Bouvet M, Singh SR, Tsuchiya H and Hoffman RM: Trabectedin and irinotecan combination regresses a cisplatinum-resistant osteosarcoma in a patient-derived orthotopic xenograft nudemouse model. Biochem Biophys Res Commun 513(2): 326-331, 2019. PMID: 30955860. DOI: 10.1016/j.bbrc.2019.03.191

11 Igarashi K, Kawaguchi K, Kiyuna T, Miyake K, Miyake M, Li Y, Nelson SD, Dry SM, Singh AS, Elliott IA, Russell TA, Eckardt MA, Yamamoto N, Hayashi K, Kimura H, Miwa S, Tsuchiya H, Eilber FC and Hoffman RM: Temozolomide regresses a doxorubicin-resistant undifferentiated spindle-cell sarcoma patient-derived orthotopic xenograft (PDOX): precision-oncology nude-mouse model matching the patient with effective therapy. J Cell Biochem 119(8): 6598-6603, 2018. PMID: 29737543. DOI: 10.1002/jcb.26792

12 Igarashi K, Kawaguchi K, Murakami T, Kiyuna T, Miyake K, Nelson SD, Dry SM, Li Y, Yanagawa J, Russell TA, Singh AS, Yamamoto N, Hayashi K, Kimura H, Miwa S, Tsuchiya H, Eilber FC and Hoffman RM: Intra-arterial administration of tumor-targeting Salmonella typhimurium A1-R regresses a cisplatin-resistant relapsed osteosarcoma in a patient-derived orthotopic xenograft (PDOX) mouse model. Cell Cycle 16(12): 1164-1170, 2017. PMID: 28494180. DOI: 10.1080/15384101. 2017.1317417

13 Igarashi K, Kawaguchi K, Kiyuna T, Miyake K, Miyake M, Li Y, Nelson SD, Dry SM, Singh AS, Elliott IA, Russell TA, Eckardt MA, Yamamoto N, Hayashi K, Kimura H, Miwa S, Tsuchiya H, Eilber FC and Hoffman RM: Temozolomide combined with irinotecan regresses a cisplatinum-resistant relapsed osteosarcoma in a patient-derived orthotopic xenograft (PDOX) precision-oncology mouse model. Oncotarget 9(8): 7774-7781, 2018. PMID: 29487690. DOI: 10.18632/oncotarget. 22892

14 Zhou Y, Shen JK, Yu Z, Hornicek FJ, Kan Q and Duan Z: Expression and therapeutic implications of cyclin-dependent kinase 4 (CDK4) in osteosarcoma. Biochim Biophys Acta Mol Basis Dis 1864(5 Pt A): 1573-1582, 2018. PMID: 29452249. DOI: $10.1016 /$ j.bbadis.2018.02.004

15 Igarashi K, Kawaguchi K, Kiyuna T, Murakami T, Miwa S, Nelson SD, Dry SM, Li Y, Singh A, Kimura H, Hayashi K, Yamamoto N, Tsuchiya H, Eilber FC and Hoffman RM: Patient-derived orthotopic xenograft (PDOX) mouse model of adult rhabdomyosarcoma invades and recurs after resection in contrast to the subcutaneous ectopic model. Cell Ccycle 16(1): 91-94, 2017. PMID: 27830986. DOI: 10.1080/15384101. 2016.1252885

16 Hiroshima Y, Maawy A, Zhang Y, Zhang N, Murakami T, Chishima T, Tanaka K, Ichikawa Y, Bouvet M, Endo I and Hoffman RM: Patient-derived mouse models of cancer need to be orthotopic in order to evaluate targeted anti-metastatic therapy. Oncotarget 7(44): 71696-71702, 2016. PMID: 277659 34. DOI: 10.18632 /oncotarget.12322

17 Yamamoto M, Zhao M, Hiroshima Y, Zhang Y, Shurell E, Eilber FC, Bouvet M, Noda M and Hoffman RM: Efficacy of tumortargeting Salmonella A1-R on a melanoma patient-derived orthotopic xenograft (PDOX) nude-mouse model. PloS One 
11(8): e0160882, 2016. PMID: 27500926. DOI: 10.1371/journal. pone. 0160882

18 Igarashi K, Kawaguchi K, Li S, Han Q, Tan Y, Murakami T, Kiyuna T, Miyake K, Miyake M, Singh AS, Eckardt MA, Nelson SD, Russell TA, Dry SM, Li Y, Yamamoto N, Hayashi K, Kimura H, Miwa S, Tsuchiya H, Singh SR, Eilber FC and Hoffman RM: Recombinant methioninase in combination with doxorubicin (DOX) overcomes first-line DOX resistance in a patient-derived orthotopic xenograft nude-mouse model of undifferentiated spindle-cell sarcoma. Cancer Lett 417: 168173, 2018. PMID: 29306021. DOI: 10.1016/j.canlet.2017. 12.028
19 Higuchi T, Miyake K, Sugisawa N, Oshiro H, Zhang Z, Razmjooei S, Yamamoto N, Hayashi K, Kimura H, Miwa S, Igarashi K, Bouvet M, Singh SR, Tsuchiya H and Hoffman RM: Olaratumab combined with doxorubicin and ifosfamide overcomes individual doxorubicin and olaratumab resistance of an undifferentiated softtissue sarcoma in a PDOX mouse model. Cancer Lett 451: 122-127, 2019. PMID: 30867142. DOI: 10.1016/j.canlet.2019.03.003

Received June 6, 2019

Revised June 28, 2019

Accepted July 1, 2019 